\title{
Prof. Dr. José Miguel Barea Navarro
}

\section{June 1942-03. April 2018}

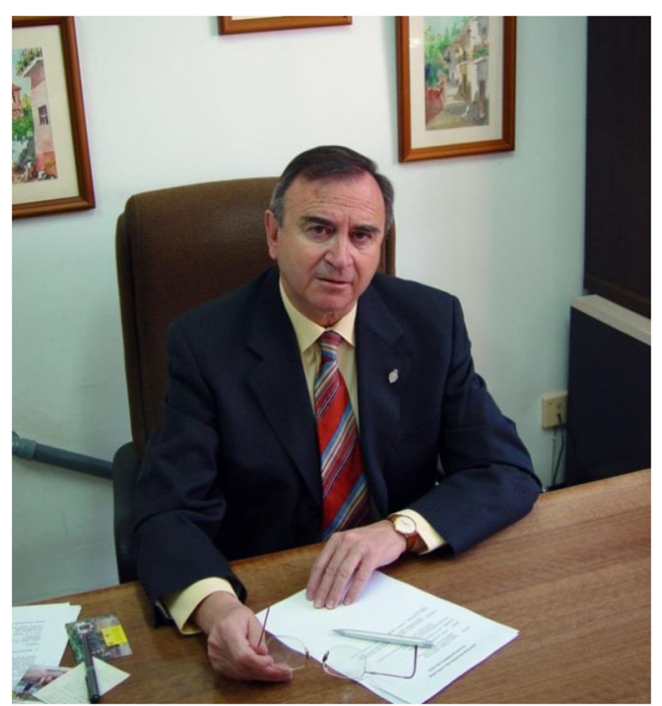

José Miguel Barea was Research Professor at the Estación Experimental del Zaidín (EEZ-CSIC, Granada, Spain). He devoted his entire professional career in the field of beneficial plant - microbe interactions and, more specifically, in the study of arbuscular mycorrhizas. He was a pioneer in Spain in mycorrhiza research and one of the pioneers in Europe. Most of the researchers who study mycorrhiza in Spain and in many Ibero-American countries have been trained under his supervision. He will be remembered for his contributions to the scientific knowledge of arbuscular mycorrhizas and their interactions with rhizosphere microorganisms.

Throughout his long scientific career, José Miguel Barea supervised $32 \mathrm{Ph}$. D. theses, published more than 170 scientific papers and about one hundred book chapters. He led multiple research projects. His way of understanding science, always open to sharing his knowledge and exploring new possibilities, led José Miguel to collaborate with researchers from the entire world. His links with other European researchers were particularly strong and led to a great development of mycorrhiza research in Europe.

Apart from his scientific contributions, it is important to highlight his great human quality. José Miguel Barea was an extremely generous person. The doors of his office were always open for any consultation, and his laboratories and equipment available to researchers from any part of the world. One of the reasons for his success was his extraordinary intelligence and his enormous capacity for work. But that enthusiasm for his work was compatible with a great vitality and ability to enjoy life. He traveled a lot and wherever he went, he left friends and disciples, who remember fondly the excellent teacher and friend he was.

\section{Azcón-Aguilar}

Estación Experimental del Zaidín (CSIC)

18008 Granada, Spain 